\title{
A study on Vulvovaginal candidiasis among non-pregnant women
}

\author{
Kavitha. $\mathbf{Y}^{1}$, Mohan $\mathrm{S}^{2}$, Anandi $\mathbf{V}^{3}$, Babu $\mathbf{H}^{4}$ \\ ${ }^{1}$ Dr. Kavitha.Y, Associate Professor, ${ }^{2}$ Dr. Mohan S, Senior Associate Professor, ${ }^{3}$ Dr. Anandi V, Professor, These three \\ authors are affiliated with Department of Microbiology, Vinayaka Missions Medical College \& Hospital, Karaikal- \\ 609609, Vinayaka missions research foundation (Deemed to be University) ${ }^{4}$ Dr. Harish Babu, Assistant Professor, \\ Department of Obstetrics and Gynecology, Vinayaka Missions Medical College \& Hospital, Karaikal- 609609 Vinayaka \\ missions research foundation (Deemed to be University), Karaikal- 609609 Puducherry, India
}

Corresponding Author: Dr. Mohan S, Address- Department of Microbiology, VMMC \& H, Karaikal, Vinayaka Missions Research Foundation (Deemed to be University). Karaikal- 609609, Puducherry, India. E-mail Address: drmohanmmc@gmail.com

\begin{abstract}
Introduction: Candida species are opportunistic pathogens which cause a wide variety of infections in humans ranging from trivial intertriginous infection to fatal candidemia. Vulvo vaginal candidiasis (VVC) is an acute inflammatory disease and a frequent reason for gynaecological consultations. The present study aimed at determining prevalence of Candida species from VVC and to speciate isolated Candida with antifungal susceptibility profile among non pregnant women. Materials and Methods: This was a cross sectional study conducted over a period of six monthsin the department of microbiology and Obstetrics and Gynecology. Swabs collected from patients were subjected to microscopy (Gram s stain) and culture on Sabouraud's dextrose agar. Species level was identified by using chrome agar. Antifungal susceptibility testing: Antifungal susceptibility testing was performed by NCCLS M44-A Disc diffusion method. Results: A total of 209 high vaginal swabs were collected from non pregnant women. 71 patients high vaginal swabs yielded growth of Candida species and accounted for 33.97\%. Most commonly isolated species were Candida albicans 31 (43.66\%) followed by Candida tropicalis $27(38.03 \%)$ and Candida glabrata $13(18.31 \%)$. All species were found to be susceptible to Amphotericin B. Among commonly used antifungals, clotrimazole was found to be most susceptible. But, C.glabrata species was least susceptible to frequently used antifungals. Conclusion: Candida albicans was the most commonly isolated species. CHRO Magar is rapid, technically simple and cost effective compared to time consuming technically demanding expensive conventional methods. Performing antifungal susceptibility is useful in choosing appropriate antifungal in treating vulvo vaginal candidiasis.
\end{abstract}

Key words: Vulvo vaginal candidiasis, Candida albicans, CHROM agar

\section{Introduction}

Vulvo vaginal candidiasis (VVC) is an acute inflammatory disease and a frequent reason for gynecological consultation as it can affect up to $75 \%$ of women of child-bearing age [1]. Clinical signs and symptoms include intense pruritus, vaginal discharge, anerythematous vulva and dyspareunia [2]. Until recently, the problem of vaginal candidiasis was often ignored, or treated as an insignificant problem forthe female population. It received more focus onlyafter Herman Gardner said: "Vaginitis can cause morein convenience than any other gynaecological disease. In addition, many mental and emotional problems are associated with vaginitis "[3].

Manuscript received: $14^{\text {th }}$ August 2018

Reviewed: $24^{\text {th }}$ August 2018

Author Corrected: $30^{\text {th }}$ August 2018

Accepted for Publication: $3^{\text {rd }}$ September 2018
There are many risk factors for development of vulvo vaginal candidiasis, like vaginal ecosystem, pregnancy, hormonal contraception, diabetes, stress recent antibioticuse, dietary practices, gastrointestinal colonization by theorganism, clothing and weaken immune-compromised system [4].

VVC is most often caused byCandida albicans, however, other species of Candidasuch as Candida glabrata, Candida parapsilosis, and Candida tropicalis are emerging [5]. Azole antifungals are being frequently being used to treat infections caused by Candida species Recently studies reported that intrinsic azole resistance in some Candida species as well as development of high-level azole resistance is a problem of critical importance in the clinical setting [6]. Mostof the studies done previously focused on immunocompromised 


\section{Original Research Article}

subjects, especially pregnant women, with few studies on other wise immunocompetent women.

The present study aimed at determining prevalence of Candida species from VVC and to speciate isolated Candida with antifungal susceptibility profile among non pregnant women.

\section{Material and Methods}

This was a cross sectional study conducted over a period of six months in the departmentof microbiology in collaboration with the department of Obstetrics and Gynaecology of Vinayaka mission's medical college and hospital. Study population consisted of non pregnant women attending OPD of Obstetrics and Gynaecology. Verbal consent was obtained from the women before sample collection. Clinical examination was performed of each participant and recorded signs of vaginal abnormalities. A pair of highvaginal swabs were obtained from the posterior vaginal fornix of the subjects aseptically with the help of a vaginal speculum. Swabs collected from patients were subjected to microscopy (Gram s stain) and culture on Sabouraud's dextrose agar.

Species identification: CHRO Magar was used for preliminary identification of yeast and to detect mixed infections. Candida species were differentiated by colonial morphology and colors, which were generated by a chromogenic in the agar, asdescribed by Wortman et al [7].

Antifungal susceptibility testing: Antifungal susceptibility testing was performed by NCCLS M44 - A Disc diffusion method.
Inoculum was prepared by picking five distinct colonies of approximately $1 \mathrm{~mm}$ in diameter from $24 \mathrm{~h}$ old culture of Candida species. Colonies were suspended in $5 \mathrm{ml}$ of sterile saline and its turbidity was adjusted visually with the transmittance to that produced by a 0.5 McFarland standard. Inoculation of test plates were done with a sterile cotton swab dipped into the suspension. The dried surface of a sterile MuellerHinton + GMB (glucose and methylene blue) agar plate was inoculated by evenly streaking the swab over the entire agar surface. Anti fungal disks were dispensed onto the surface of inoculated agar plate. Plates were incubated at $35^{\circ} \mathrm{C}$ and examined after $20-24$ hours. The zone of inhibition was measured and the results were recorded as susceptible/resistant [8]. Patients aged between 15-45 years and non-pregnant were included in the study. Immunocompromised patients, menstruating women and patients who were taken antifungal therapy in past two weeks (before sample collection) were excluded. Statistical analysis was done by simple percentage method.

\section{Results}

A total of 209 high vaginal swabs were collected from non pregnant women aged between 15-45 years attending with any vulvovaginal candidiasis symptoms (itching, soreness, vaginal secretions, inflammation, rashes etc.). Of the 209 patients included in the study, 71 patients high vaginal swabs yielded growth of Candida species and accounted for $33.97 \%$. Majority of the patients yielded growth were ranged between 26-35 years and accounted for $54 \%$ followed by $16-25$ years which showed $30 \%$. Least growth was seen in the patients between the age group of 36-45 years which accounted for $14 \%$ (Table. 1 ).

Table-1: Age wise distribution of Candida growth on SDA medium.

\begin{tabular}{|c|c|}
\hline Age & Growth on SDA \\
\hline $15-25$ & $22(30 \%)$ \\
\hline $26-35$ & $39(54 \%)$ \\
\hline $36-45$ & $10(14 \%)$ \\
\hline
\end{tabular}

Table-2: Candida species isolated from high vaginal swabs.

\begin{tabular}{|c|c|}
\hline Candida species & Number (\%) \\
\hline Candida albicans & $31(43.66 \%)$ \\
\hline Candida tropicalis & $27(38.03 \%)$ \\
\hline Candida glabrata & $13(18.31 \%)$ \\
\hline Total & $71(100 \%)$ \\
\hline
\end{tabular}

Most commonly isolated species were Candida albicans 31 (43.66\%) followed by Candida tropicalis 27(38.03\%) and Candida glabrata 13(18.31\%) (Table.2) 
Original Research Article

Table-3: Antifungal susceptibility of Candida species isolated.

\begin{tabular}{|c|c|c|c|c|}
\hline Candida species & Clotrimazole & Fluconazole & Miconazole & Amphotericin B \\
\hline Candida albicans $(\mathrm{n}=31)$ & $22(70.96 \%)$ & $11(35.48 \%)$ & $19(61.29 \%)$ & $100(100 \%)$ \\
\hline Candida tropicalis $(\mathrm{n}=27)$ & $15(55.55 \%)$ & $7(25.92 \%)$ & $13(48.14 \%)$ & $100(100 \%)$ \\
\hline Candida glabrata $(\mathrm{n}=13)$ & $3(23.07 \%)$ & $3(23.07 \%)$ & $5(38.46 \%)$ & $100(100 \%)$ \\
\hline
\end{tabular}

\section{Discussion}

In the present study infection rate of vulvovaginal candidiasis was found to be $33.97 \%$. But according to the study conducted by Enweani et al [9] V VC in nonpregnant women was $40.6 \%$ which is high compared to the present study. Eckert et al [10] found $25 \%$ of VVC among non pregnant women. The prevalence of vaginal candidiasis reported by different studies was $16.5 \%$, $21.31 \%$, and $19 \%[11,12,13]$. This relatively low prevalence of vaginal candidiasis among women in other studies probably due to to adequate knowledge, good personal hygiene, and normal levels of estrogens and corticoids [14].

In the present study, Candida species was predominantly isolated in the age group of 26-35 years which accounted for $54 \%$. This may be due to high sexual activity, poor personal hygiene, the use of contraceptives, and drug abuse among this age-group. Alo et al[15] reported a higher prevalence of $C$. albicans (33.33\%) within the age bracket of 36-40 years, while those between 20 and 25 years had the lowest prevalence $(20.42 \%)$.

In the present study, low Candida infection was found in age group 36-45 years which accounted for 14\%. This finding is in line with a previous report by Okungbowa et al[16] who reported prevalence of $10 \%$ and $2 \%$ within the age-groups of $36-45$ and over 46 years, respectively. They reported that this was probably due to the possible increase in vaginal immunity with age as they have decreased levels of estrogen and corticoids, and thus are resistant to Candida infections.

In the present study, Candida albicans $31 \quad$ (43.66\%) was the most predominant species isolated followed by Candida tropicalis $27(38.03 \%)$ and Candida glabrata $13(18.31 \%)$, similar to other study by Grigoriou et al, the most commonly isolated species from the patients was C. albicans.

According to Habibipour R, [17]C. albicans (81.3\%) predominantly and then $C$. glabrata $(11 \%), C$. tropicalis $(4.4 \%)$ and $C$. kuresi $(2.2 \%)$ were isolated from VVC. A similar distribution of Candida spp. Similar observation was found by other authors $[18,19]$.
C. albicans adheres to vaginal epithelial cells in significantly higher numbers than do other Candida species [20]. Vaginitis induced by non-albicans species is clinically indistinguishable from that caused by $C$. albicans [21]. The reason for the increase in incidence of VVC caused by non-albicans species is thought to be single-dose antifungal treatment, low-dosage azolemaintenance regimens, and the use of over-the-counter antimycotics[22].

In the present study, all three species of Candida were least susceptible to fluconazole and no Candida species showed resistance to amphotericin B. Few strains of Candida species exhibited multi drug resistance to routinely used antifungals. Relatively low levels of fluconazole resistance were observed in all species of Candida isolated.Resistance to fluconazole is of great concern as it is the most common azole used for the treatment of candidiasis including VVC. Fluconazole is available in both intravenous and oral formulations with high bioavailability and is more cost-effective than other antifungal agents.

As per study by Mishra et al[23] all C. glabrata, 50\% C. tropicalis and $12 \%$ C. albicans isolates were found to be resistant to fluconazole. But, another study by Khan $\mathrm{M}$ et al observed, C. krusei showed 100\% resistance to fluconazole [24]. It is worth mentioning that the non-albicans species had the highest levels of resistance compared to C. albicans. C. glabrata was the least susceptible to the tested antifungals. Although amphotericin B iseffective against most strains of Candida spp., its usage is limited due to the nephro toxicity associated with it.

\section{Conclusion}

Candida albicans was the most commonly isolated species followed by C.tropicalis. Among commonly used antifungals, clotrimazole was found to be most susceptible. But, C.glabrata species was least susceptible to frequently used antifungals. Hence, species level identification is required to choose appropriate antifungal and chrom agar medium will helpful to mycology laboratories for rapid identification of clinically important candida spp. 


\section{Original Research Article}

\section{Contributions by Authors}

1. Dr. Harish Babu: Clinical examination of patients and specimen collection.

2. Dr. Kavita: Study design and microbiology work up.

3. Dr. Anandi and Dr. Mohan: Collection of review literature and manuscript preparation.

Information added to existing knowledge: Usually involvement of $\mathrm{C}$. albicans is frequent in causing vulvovaginal candidiasis and other species cause vulvovaginal candidiasis infrequently. But in our study, isolation rate of C.tropicalis is almost equal to C.albicans.

Funding: Nil, Conflict of interest: None initiated Permission from IRB: Yes

\section{References}

1. Sobel JD. Pathogenesis and treatment of recurrent vulvovaginal candidiasis. Clin Infect Dis. 1992 Mar;14 Suppl 1:S148-53.

2. Borges S, Silva J, Teixeira P. The role of lactobacilli and probiotics in maintaining vaginal health. Arch Gynecol Obstet. 2014 Mar;289(3):479-89. doi: 10.1007/ s 00404 -013-3064-9. Epub 2013 Oct 30.

3. Sobel JD, Faro S, Force RW, et al. Vulvovaginal candidiasis: epidemiologic, diagnostic, and therapeutic considerations. Am J Obstet Gynecol. 1998 Feb;178 (2) : 203-11.

4. Ahmad A, Khan AU. Prevalence of Candida species and potential risk factors for vulvovaginal candidiasis in Aligarh, India. Eur J Obstet Gynecol Reprod Biol. 2009 May; 144(1):68-71. doi: 10.1016/j.ejogrb.2008.12.020. Epub 2009 Mar 3.

5. Jindal N, Gill P, Aggarwal A. An epidemiological study of vulvovaginal candidiasis in women of childbearing age. Indian J Med Microbiol. 2007 Apr;25 (2): 175-6.

6. Flowers SA, Colón B, Whaley SG, et al. Contribution of clinically derived mutations in ERG11 to azole resistance in Candida albicans. Antimicrob Agents Chemother. 2015 Jan;59(1):450-60. doi: 10.1128/AAC. 03470-14. Epub 2014 Nov 10.

7. Wortman JR, Gilsenan JM, Joardar V, The 2008 update of the Aspergillus nidulans genome annotation: a community effort. Fungal Genet Biol. 2009 Mar;46 Suppl 1:S2-13. doi: 10.1016/j.fgb.2008.12.003. Epub 2008 Dec 25.
8. Wayne, P.A.: Method for Antifungal Disk Diffusion Susceptibility Testing of Yeasts, NCCLS document M44 -A (2004).

9. Enweani IB, Gugnani HC, Okobia R, Ojo SB. Effect of contraceptives on the prevalence of vaginal colonization with Candida species in Edo State, Nigeria. Rev IberoamMicol. 2001 Dec;18(4):171-3.

10. Eckert LO, Hawes SE, Stevens CE, et al. Vulvovaginal candidiasis: clinical manifestations, risk factors, management algorithm. Obstet Gynecol. 1998 Nov;92 (5): 757-65.

11. Mahadani JW, Dekate RR, Shrikhande AV. Cytodiagnosis of discharge per vaginum. Indian $\mathrm{J}$ Pathol Microbiol. 1998 Oct;41(4):403-11.

12. Nandan D, Gupta YP, Krishnan V, et al. Reproductive tract infection in women of reproductive age group in Sitapur/Shahjahanpur District of Uttar Pradesh. Indian J Public Health. 2001 Jan-Mar;45(1):8-13.

13. Aring BJ, Mankodi PJ, Jasani JH. Incidence of vaginal candidiasis in leucorrhoea in women attending in OPD of gynecology and obstetrics department. Int J Biomed Adv Res. 2012;3(12):867-869.

14. Fernández Limia O, Lantero MI, Betancourt A, et al. Prevalence of Candida albicans and Trichomonas vaginalis in pregnant women in Havana City by an immunologic latex agglutination test. MedGen Med. 2004 Oct 15;6(4):50.

15. Alo MN, Anyim C, Onyebuchi AK, Okonkwo EC. Prevalence of asymptomatic co-Infection of candidiasis and vaginal trichomoniasis among pregnant women in Abakaliki, South-Eastern Nigeria. J Nat Sci Res. 2012; 2 (7): 87-91.

16. Okungbowa FI, Isikhuemhen OS, Dede AP. The distribution frequency of Candida species in the genitourinary tract among symptomatic Individuals in Nigerian cities.Rev IberoamMicol.2003 Jun;20(2):60-3

17. Habibipour R. Prevalence Rate of Vulvovaginal Candidiasis and Identification of Candida Species in Women in Referred to Hamedan Hospitals 2013-2014, Westof Iran. Zahedan J Res Med Sci. 2013; 18(3):6250.

18. Corsello S, Spinillo A, Osnengo G, et al. An epidemiological survey of vulvovaginal candidiasis in Italy. Eur J ObstetGynecolReprod Biol. 2003 Sep 10;110(1):66-72. 


\section{Original Research Article}

19. Nazeri M, Mesdaghinia E, Moravej SAR, Atabakhshiyan R, SoleymaniF. Prevalence of vulvovaginal candidiasis and frequency of Candidaspecies in women [in Persian]. J Mazandaran Univ Med Sci.2012; 21 (86): 254-62.

20. Simões JA, Giraldo PC, Faúndes A. Prevalence of cervicovaginal infections during gestation and accuracy of clinical diagnosis. DOI: 10. 1155/ S106474 4998000246

21. Bauters TG, Dhont MA, Temmerman MI, Nelis HJ. Prevalence of vulvovaginal candidiasis and susceptibility to fluconazole in women. Am J Obstet Gynecol. 2002 Sep;187(3):569-74.
22. Reed BD. Risk factors for Candida vulvovaginitis. Obstet Gynecol Surv. 1992 Aug; 47(8):551-60.

23. Mishra M, Agrawal S, Raut S, et al. Profile of yeasts isolated from urinary tracts of catheterized patients. J Clin Diagn Res. 2014 Feb; 8 (2): 44-6. doi: 10. 7860/ JCDR/ 2014/ 6614. 4003. Epub 2014 Feb 3.

24. Khan M, Ahmed J, Gul A, Ikram A, Lalani FK. Antifungal susceptibility testing of vulvovaginal Candida species among women attending antenatal clinic in tertiary care hospitals of Peshawa. 2018; 11: 447-56.

\section{How to cite this article?}

Kavitha. Y, Mohan S, Anandi V, Babu H. A study on Vulvovaginal candidiasis among non-pregnant women. Trop J Path Micro 2018; 4(5):396-400.doi:10.17511/jopm.2018.i05.05 